\title{
Neurofilaments Switch between Distinct Mobile and Stationary States during Their Transport along Axons
}

\author{
Niraj Trivedi, ${ }^{1}$ Peter Jung, ${ }^{2}$ and Anthony Brown ${ }^{1}$ \\ ${ }^{1}$ Center for Molecular Neurobiology and Department of Neuroscience, The Ohio State University, Columbus, Ohio 43210, and ${ }^{2}$ Department of Physics and \\ Astronomy, Ohio University, Athens, Ohio 45701
}

\begin{abstract}
We have developed a novel pulse-escape fluorescence photoactivation technique to investigate the long-term pausing behavior of axonal neurofilaments. Cultured sympathetic neurons expressing a photoactivatable green fluorescent neurofilament fusion protein were illuminated with violet light in a short segment of axon to create a pulse of fluorescent neurofilaments. Neurofilaments departed from the photoactivated regions at rapid velocities, but the overall loss of fluorescence was slow because many of the neurofilaments paused for long periods of time before moving. The frequency of neurofilament departure was more rapid initially and slower at later times, resulting in biphasic decay kinetics. By computational simulation of the kinetics, we show that the neurofilaments switched between two distinct states: a mobile state characterized by intermittent movements and short pauses (average $=30 \mathrm{~s}$ ) and a stationary state characterized by remarkably long pauses (average $=60 \mathrm{~min}$ ). On average, the neurofilaments spent $92 \%$ of their time in the stationary state. Combining short and long pauses, they paused for $97 \%$ of the time, resulting in an average transport rate of $0.5 \mathrm{~mm} / \mathrm{d}$. We speculate that the relative proportion of the time that neurofilaments spend in the stationary state may be a principal determinant of their transport rate and distribution along axons, and a potential target of mechanisms that lead to abnormal neurofilament accumulations in disease.
\end{abstract}

Key words: axonal transport; photoactivation; green fluorescent protein; neurofilament; culture; cytoskeleton

\section{Introduction}

Long-term tracking of neurofilament protein transport in vivo on a time scale of days or weeks using radioisotopic pulse labeling has demonstrated that neurofilaments move slowly along axons in an anterograde direction at average rates of $\sim 0.1-3 \mathrm{~mm} / \mathrm{d}$ (i.e., 0.001-0.03 $\mu \mathrm{m} / \mathrm{s}$ ) (Nixon, 1991; Lasek et al., 1992). In contrast, short-term tracking of single neurofilaments in cultured neurons on a time scale of seconds or minutes using live-cell fluorescence imaging has demonstrated that these cytoskeletal polymers actually move at fast rates, which average $\sim 0.4-0.6 \mu \mathrm{m} / \mathrm{s}$, and that their movements are both bidirectional and intermittent (Roy et al., 2000; Wang et al., 2000; Wang and Brown, 2001; Ackerley et al., 2003; Uchida and Brown, 2004). To reconcile these observations, we have proposed that neurofilaments move rapidly in vivo and that their overall velocity is slow because they spend most of the time pausing (Brown, 2000, 2003b). We refer to this as the "stop-and-go" hypothesis.

To investigate whether the stop-and-go hypothesis can explain the kinetics of slow axonal transport in vivo, we used computational modeling to simulate the movement of neurofilaments in a radioisotopic pulse-labeling experiment (Brown et al., 2005; Craciun et al., 2005). The kinetic parameters of the model

Received Sept. 27, 2006; revised Nov. 20, 2006; accepted Dec. 4, 2006.

This work was funded by National Institutes of Health Grant NS-38526 (A.B.). We thank George Patterson for the PPA-GFP-C1 vector, Virginia Lee for the NFL polyclonal antibody, and Kitty Jensen in the Brown laboratory for cloning mouse NFL.

Correspondence should be addressed to Dr. Anthony Brown, Center for Molecular Neurobiology, Rightmire Hall, The Ohio State University, 1060 Carmack Road, Columbus, OH 43210. E-mail: brown.2302@osu.edu. DOI:10.1523/JNEUROSCI.4227-06.2007

Copyright $\odot 2007$ Society for Neuroscience $\quad$ 0270-6474/07/270507-10\$15.00/0 were based on our detailed measurements of the short-term moving and pausing behavior of neurofilaments tagged with green fluorescent protein (GFP) moving through photobleached gaps in the axonal neurofilament array of cultured neurons (Wang and Brown, 2001). Using this approach, we were not able to match the in vivo pulse-labeling kinetics; the pulse of radiolabeled neurofilaments moved too rapidly and did not spread enough (Brown et al., 2005). To match the shape, velocity, and spreading of a pulse of radiolabeled neurofilaments in vivo, we had to assume that neurofilaments can enter a second state in which they pause for much longer periods of time. Thus, we proposed that neurofilaments switch between distinct mobile and stationary states, which we termed on track and off track, respectively. According to this hypothesis, neurofilaments in the on-track state move along microtubule tracks, alternating between short bouts of rapid movement and short pauses, which correspond to the movements and pauses observed in our live-cell imaging studies. In contrast, neurofilaments in the off-track state are temporarily disengaged from their microtubule tracks and pause for long periods of time without movement (Brown et al., 2005; Craciun et al., 2005). Thus our short-term observations on moving neurofilaments in axons may have underestimated their true pausing behavior.

To test this hypothesis, we have developed a novel pulseescape fluorescence photoactivation technique for observing neurofilament transport. This technique has enabled us to investigate the long-term pausing behavior of neurofilaments in cultured neurons. Our analysis of the pulse-escape kinetics confirms the existence of distinct mobile and stationary states. By computational simulation of the experimental data, we show that the 
neurofilaments spend $\sim 92 \%$ of their time in the stationary state, pausing on average for $60 \mathrm{~min}$. Thus, the motile behavior of neurofilaments is dominated by remarkably long pauses, and this explains why the overall rate of neurofilament transport is so slow.

\section{Materials and Methods}

Plasmid constructs. Rat neurofilament protein M (NFM) cDNA was excised from the pEGFP-NFM construct of Wang et al. (2000) using $B a m \mathrm{HI}$ and $K p n \mathrm{I}$ and then subcloned into the corresponding sites of the pPA-GFP-C1 vector of Patterson and Lippincott-Schwartz (2002). The resulting PPA-GFP-NFM fusion construct encoded photoactivatable GFP (PA-GFP), which is the V163A/T203H variant of a codonoptimized wild-type GFP, connected to the N terminus of rat NFM by a 25 aa linker. The pDsRed2-C1 vector was purchased from Clontech (Mountain View, CA). The pNFL vector was constructed by cloning mouse neurofilament protein L (NFL) cDNA (GenBank accession number DQ201635) into the pEGFP-C1 vector purchased from Clontech and then excising the GFP portion. All constructs were verified by DNA sequencing.

Culture and transfection of neurons. Superior cervical ganglia (SCGs) were dissected from 2- to 3-d-old Sprague Dawley rats (Harlan, Indianapolis, IN) and dissociated as described previously (Brown, 2003a). Suspensions of cells from 7-10 ganglia were centrifuged at $100 \times g$ for 5 min at room temperature and resuspended in $100 \mu \mathrm{l}$ of mouse neuron nucleofection solution (Amaxa Biosystems, Gaithersburg, MD). The cells were cotransfected with $5 \mu \mathrm{g}$ of pPA-GFP-NFM and $5 \mu \mathrm{g}$ of pDsRed2-C1 by electroporation using program G-13 of an Amaxa Nucleofector (Amaxa Biosystems) and then diluted 1:5 with Leibovitz's L15 medium (Invitrogen, Carlsbad, CA) containing $0.5 \mathrm{mg} / \mathrm{ml} \mathrm{BSA}$ ("plating medium"). After recovery at $37^{\circ} \mathrm{C}$ for $10 \mathrm{~min}$, the cells were plated at a density of 0.2 dissociated ganglia per coverslip onto $40 \mathrm{~mm}$ round number (No.) 1.5 glass coverslips (Bioptechs, Butler, PA) that had been coated with poly-D-lysine and laminin as described previously (Brown, 2003a). After allowing the cells to attach for $2 \mathrm{~h}$ at $37^{\circ} \mathrm{C}$, the plating medium was replaced with Leibovitz's L15 medium supplemented with $0.6 \%$ (w/v) D-glucose, $2 \mathrm{~mm}$ L-glutamine, $100 \mathrm{ng} / \mathrm{ml} 2.5 \mathrm{~S}$ nerve growth factor (BD Biosciences, Bedford, MA), $10 \%$ (v/v) adult rat serum (Harlan), and 0.5\% hydroxypropyl methylcellulose (Methocel; Dow Corning, Midland, MI). The cells were maintained in this medium at $37^{\circ} \mathrm{C}$.

Culture and transfection of SW13 vim - cells. SW13 vimentin-deficient $($ vim - ) cells (Sarria et al., 1990) were grown on uncoated No. 1.5 glass coverslips in DMEM/F12 medium (Gibco Cell Culture Products; Invitrogen), supplemented with $10 \mu \mathrm{g} / \mathrm{ml}$ gentamycin and $5 \%$ (v/v) fetal calf serum, at $37^{\circ} \mathrm{C}$ in $5 \% \mathrm{CO}_{2}$. For live-cell imaging, we used $40 \mathrm{~mm}$ round coverslips (Bioptechs), and for immunostaining, we used $22 \times 22$ $\mathrm{mm}$ square coverslips. Once cells reached $\sim 50 \%$ confluence, they were transfected using Lipofectamine 2000 (Invitrogen) with $2 \mu \mathrm{g}$ of total DNA in serum-free DMEM/F12 medium (Invitrogen).

Imaging and photoactivation. For live-cell imaging, the coverslips were mounted in Hibernate-E low-fluorescence medium (Brain Bits, Springfield, IL) supplemented with $2 \%$ (v/v) B27 supplement (Invitrogen), 100 $\mathrm{ng} / \mathrm{ml} 2.5 \mathrm{~S}$ nerve growth factor, $2 \mathrm{~mm}$ L-glutamine, $0.4 \%(\mathrm{w} / \mathrm{v}$ ) D-glucose, and $37.5 \mathrm{~mm} \mathrm{NaCl}$ ("observation medium") in a Bioptechs FCS2 closedbath imaging chamber maintained at $37^{\circ} \mathrm{C}$. The objective was maintained at $37^{\circ} \mathrm{C}$ using a Bioptechs objective heater. In some experiments, 2 mM sodium iodoacetate (Sigma-Aldrich, St. Louis, MO) and $5 \mu \mathrm{m}$ carbonyl cyanide $m$-chlorophenylhydrazone (CCCP; Sigma-Aldrich) were also added to the observation medium. For these experiments, we allowed 30-60 min for the drugs to act before beginning our time-lapse imaging. All imaging was performed using a Nikon (Garden City, NY) TE2000 inverted microscope, with a standard $100 \mathrm{~W}$ mercury lamp source and a 100× Plan Apo VC 1.40 numerical aperture oil-immersion objective (Nikon). Images were acquired with a CoolSnap HQ cooled CCD camera (Roper Scientific, Trenton, NJ) and MetaMorph software (Universal Imaging, Downingtown, PA). DsRed was observed with green excitation using a tetramethylrhodamine isothiocyanate/DiI filter cube (model 41002b; Chroma Technology, Brattleboro, VT). Activated PAGFP-NFM was observed with blue excitation using an FITC/EGFP filter cube (model 41001; Chroma Technology). To minimize photobleaching, the exciting light from the mercury arc lamp was attenuated 32 -fold ( $\sim 3 \%$ light transmission) with neutral density filters. PA-GFP-NFM was activated with violet light using a violet filter cube (model 11005v2; Chroma Technology). To maximize the intensity of the exciting light for photoactivation, the neutral density filters were removed from the light path and then replaced before subsequent imaging. The camera was exposed during photoactivation, and the resulting image was used to define the location of the activated region in our subsequent analyses. These procedures were followed throughout this study.

Characterization of PA-GFP-NFM in SW13 vim - cells. To characterize PA-GFP-NFM assembly, SW13 vim - cells were transfected with pPAGFP-NFM and pNFL. One day later, the cells were fixed using $4 \%(\mathrm{w} / \mathrm{v})$ paraformaldehyde in PBS, stripped of their membranes using $0.25 \%$ (v/v) Triton X-100 in PBS, and then blocked using normal donkey serum (Jackson ImmunoResearch, West Grove, PA). NFL and PA-GFP-NFM were visualized by immunostaining using primary antibodies against NFL (rabbit polyclonal antibody NFLAS; 1:300 dilution; a gift from Dr. Virginia Lee, University of Pennsylvania, Philadelphia, PA) and NFM (mouse monoclonal antibody RMO270; 1:100 dilution; Zymed, South San Francisco, CA). The secondary antibodies were lissamine rhodamine sulfonyl chloride-conjugated donkey anti-rabbit IgG (diluted 1:100; Jackson ImmunoResearch) and Alexa 488-conjugated donkey antimouse IgG (diluted 1:200; Invitrogen). Coverslips were mounted onto slides using ProLong Antifade reagent (Invitrogen). To determine the optimal exposure time for PA-GFP-NFM activation, SW13 vim- cells were transfected with pPA-GFP-NFM, pNFL, and pDsRed2-C1. One day later, healthy well spread DsRed-positive cells were selected, and an image of the PA-GFP-NFM fluorescence was acquired to record the level of fluorescence before activation. The PA-GFP-NFM was then activated in a cumulative manner by successive $200 \mathrm{~ms}$ exposures to violet light. After each $200 \mathrm{~ms}$ exposure, an image of the PA-GFP-NFM fluorescence was acquired to visualize the level of activation.

Pulse-escape experiments. Pulse-escape experiments were performed on SCG cultures between 3 and $5 \mathrm{~d}$ after plating. Healthy DsRed-positive cells with long, smooth axons were selected, and the region to be activated was positioned in the center of the field of view. An image of the DsRed fluorescence was acquired to record the location and morphology of the axon, and an image of the PA-GFP-NFM fluorescence was acquired to establish the level of fluorescence before activation. For activation, a field-limiting diaphragm in a conjugate focal plane of the epifluorescence illumination light path was closed down to restrict the region of illumination to a circular area in the center of the field of view, thereby exposing only a short segment of the axon of interest. The activation was checked by opening the diaphragm and then taking an image of the PA-GFP-NFM fluorescence. If satisfactory, the activated region was observed by taking additional images of the PA-GFP-NFM fluorescence at $5 \mathrm{~s}, 40 \mathrm{~s}$, or $5 \mathrm{~min}$ time intervals for a maximum of 25 frames. For movies using either $40 \mathrm{~s}$ or $5 \mathrm{~min}$ intervals, the focus was checked using the DsRed fluorescence and the "show live" function in MetaMorph before acquiring each frame to compensate for any focus drift. The health and integrity of the axons was monitored between image acquisitions using differential interference contrast and the DsRed fluorescence. Any stage drift that occurred during the movie was corrected for by shifting successive images relative to each other in the $X$ and $Y$ dimensions using the "align stack" function in the MetaMorph software. Movies in which the axons were observed to move laterally or change significantly in shape were excluded from our analyses.

Quantitative analysis. For each axon, a rectangular region was drawn around the activated region in the photoactivation image, extending four to six pixels on either side of the central axis of the axon to include all of the axonal fluorescence. To calculate the background fluorescence, two adjacent rectangular regions were drawn on either side of this axonal region, each four pixels wide. The fluorescence intensity was measured for each region, and then the axonal fluorescence was corrected for background by subtracting the average background pixel intensity from the average pixel intensity in the axon, and this was repeated for each image 
in the time-lapse series. In addition, we found that we also had to account for excitation of DsRed on the PA-GFP channel. This is attributable to the broad excitation spectrum of DsRed, and it explains the faint basal level of diffuse green fluorescence observed throughout these axons before activation (see Fig. $1 C$ ) and outside of the activated regions in our movies (see Figs. 2, 3). To correct for this, we calculated a backgroundcorrected preactivation fluorescence intensity on the PA-GFP channel, and then we subtracted this intensity from the background-corrected intensities for the axon in each frame of the time-lapse series. After this correction for DsRed excitation cross talk, the length of the activated region was measured along the axis of the axon, and the corrected fluorescence intensities were expressed in analog-to-digital units per micrometer of axon $(\mathrm{ADU} / \mu \mathrm{m})$. In a few cases, a fluorescent filament moved out of the activated region before the capture of the first image in the series. When this occurred, we always knew that it had, because the time between activation and acquisition of the first image was not sufficient for even the fastest neurofilament to exit the field of view. To correct for these early departures, we measured the fluorescence intensity of the escaped filament as described above and then added it back to the corrected fluorescence intensity of the activated region, thereby providing us with the true intensity of the activated region at time 0 . For images that were visibly out of focus, we estimated the fluorescence intensity for that time interval by linear interpolation between the adjacent time intervals in the series. If the first or last image in the series was out of focus, the series was discarded. Fluorescence intensities in SW13 cells were quantified in a similar manner, except that the measurement regions were polygonal in shape and were drawn to trace the outline the entire cell, and the background regions were four to six pixels wide and were drawn to encircle the cell.

Computational modeling. The movement of photoactivated fluorescent neurofilaments in the pulse-escape experiments was simulated using the computational model described by Brown et al. (2005). The neurofilaments were treated as a uniform population aligned parallel to the long axis of the axon in a continuous overlapping array. Neurofilament length was assumed to be $4.5 \mu \mathrm{m}$, based on our published measurements of neurofilaments moving through photobleached gaps (Wang and Brown, 2001), and the neurofilaments were considered to move in either an anterograde or retrograde direction. The frequency of reversals was given by the rate constants $k_{\mathrm{ar}}$ (rate of switching from anterograde to retrograde) and $k_{\text {ra }}$ (rate of switching from retrograde to anterograde). We also defined an overall reversal rate constant, $k_{\mathrm{rev}}$, where $k_{\mathrm{rev}}=k_{\mathrm{ar}}+$ $k_{\mathrm{ra}}$. To explain the moving and pausing behavior of neurofilaments, we assumed that both anterogradely and retrogradely moving neurofilaments can engage with their tracks and exhibit bouts of rapid movement interrupted by brief pauses or disengage from their tracks and pause for more prolonged periods. We refer to these mobile and stationary states as on track and off track, respectively. The rates of switching on and off track were described by the rate constants $k_{\text {on }}$ and $k_{\text {off }}$, respectively. The simulations were all performed using a fixed $4.73 \mathrm{~s}$ time interval (Brown et al., 2005), so we express the rate constants as events per time interval rather than per second throughout this manuscript. Neurofilaments that were on track were considered to move and pause according to a matrix of transition probabilities extracted from our published experimental data (Brown et al., 2005). The overall reversal frequency $k_{\text {rev }}$ was estimated to be 0.001 , based on our published experimental data on neurofilament reversals (Uchida and Brown, 2004). Knowing that the ratio of anterograde to retrograde movements is $69 / 31$ in cultured neonatal rat SCG neurons (Wang and Brown, 2001), this means that $k_{\mathrm{ar}}=0.00031$ and $k_{\text {ra }}=0.00069$ (Brown et al., 2005). Note, however, that the ratio $k_{\mathrm{ar}} / k_{\mathrm{ra}}$ has no effect on our simulations, because we tracked the rate of neurofilament departure without regard to the direction of movement.

To equilibrate the spatial and velocity distributions of the neurofilaments within the axon before the start of our pulse-escape simulation, we initially placed all of them at some arbitrary location within a $100 \mu \mathrm{m}$ segment of axon and assigned them to be either anterograde or retrograde and pausing either on or off track in relative proportions dictated by the rate constants $k_{\text {on }}, k_{\text {off }}, k_{\mathrm{ar}}$, and $k_{\mathrm{ra}}$, as described previously (Brown et al., 2005). We then ran the simulation iteratively for some time until the neurofilaments became uniformly distributed along the axon and attained a stable distribution of velocities as dictated by the transition probability matrix. To simulate the entry and exit of neurofilaments from proximal and distal ends of the axonal segment during this equilibration phase of the simulation, neurofilaments that reached either end $(x=0$ or $100 \mu \mathrm{m})$ were instantly fed back into the axon at the opposite end, which is known as a periodic boundary condition. The result using this approach is identical to assuming an open steady-state system in which neurofilaments enter and exit at a constant rate.

After equilibration, a window was identified within the axon, and the behavior of the neurofilaments or parts of neurofilaments that lay within that window was tracked over time to simulate the behavior of fluorescent neurofilaments as they moved out of the activated region. The length of the window was taken to be $22.5 \mu \mathrm{m}$, which is the average length of the photoactivated regions for the axons that we analyzed in this study (see Results). The total neurofilament polymer length in the window was recorded as a function of time. For this portion of the simulation, the periodic boundary condition was removed, and we continued to track neurofilaments that departed from either end of the $100 \mu \mathrm{m}$ axonal segment to allow for the rare possibility of a filament reversing and moving back into the activated region. To extract the parameters $k_{\text {on }}$ and $k_{\text {off }}$ from the experimental data, we systematically varied their values to obtain the best possible match to the pulse-escape kinetics.

\section{Results}

\section{Assembly of PA-GFP-NFM into neurofilaments}

To investigate the long-term pausing behavior of neurofilaments, we created a photoactivatable neurofilament protein (PA-GFPNFM) by fusing photoactivatable GFP to the N terminus of neurofilament protein $\mathrm{M}$. To test whether the fusion protein was capable of forming neurofilaments, we cotransfected SW13 vim - cells, which lack endogenous cytoplasmic intermediate filament proteins, with NFL and PA-GFP-NFM expression constructs and then visualized the expressed proteins by immunofluorescence microscopy (Fig. 1A). NFL and PA-GFP-NFM coassembled fully into neurofilaments, and these filaments contained both proteins along their entire length, consistent with previous observations that neurofilaments are obligate heteropolymers in vivo (Ching and Liem, 1993; Lee et al., 1993). Thus, fusion of PA-GFP to the N terminus of NFM does not interfere with the ability of NFM to assemble into neurofilaments.

\section{Photoactivation of PA-GFP-NFM fluorescence}

To characterize the photoactivation of the PA-GFP-NFM fusion protein, we cotransfected SW13 vim - cells with PA-GFP-NFM, NFL, and DsRed constructs. The DsRed served as a diffusible marker and permitted us to detect the transfected cells before activation of the PA-GFP fluorescence. The PA-GFP fluorescence was efficiently activated by exposure to violet light, using the mercury arc lamp as a light source. On average, optimal activation was achieved with an exposure of $1000 \mathrm{~ms}$ (Fig. 1 B). Longer exposures resulted in less efficient activation, presumably because of photobleaching associated with excitation of the PAGFP with violet light. Based on these data, all subsequent experiments were performed using a $1000 \mathrm{~ms}$ activation time.

To use the PA-GFP-NFM fusion protein to investigate neurofilament transport, we transfected dissociated neurons from the SCGs of neonatal rats with PA-GFP-NFM and DsRed. Three to five days later, transfected neurons were identified by their DsRed fluorescence, and a short segment of axon $\sim 20 \mu \mathrm{m}$ in length was activated by exposure to violet light for $1000 \mathrm{~ms}$ (Fig. $1 C)$. In most cases, we observed good activation. When poor activation was obtained, it was most probably caused by low PAGFP-NFM expression in that neuron. In some cases, part of the selected region was activated, and part was not, most likely be- 


\section{A. PA-GFP-NFM assembles into neurofilaments}

(i) NFL

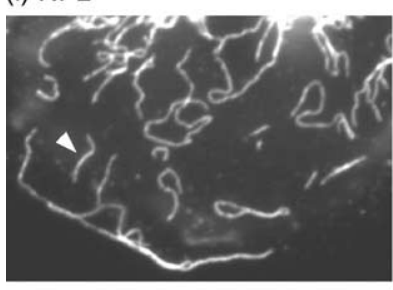

(ii) PA-GFP-NFM

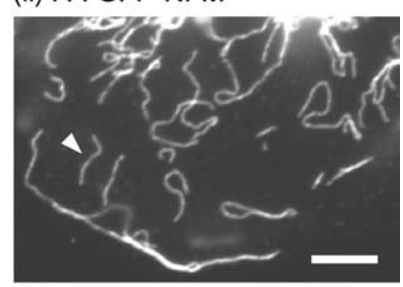

B. Time course of PA-GFP-NFM activation

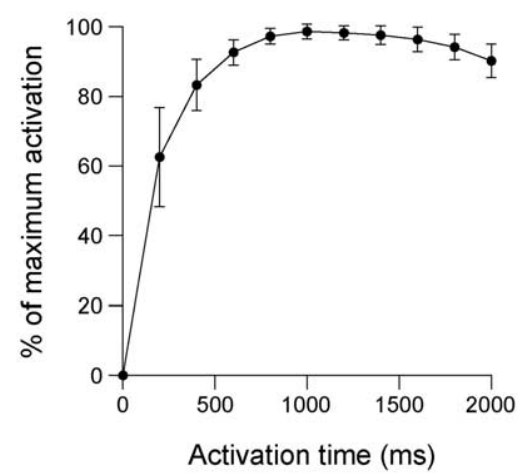

\section{PA-GFP-NFM activation in an axon}
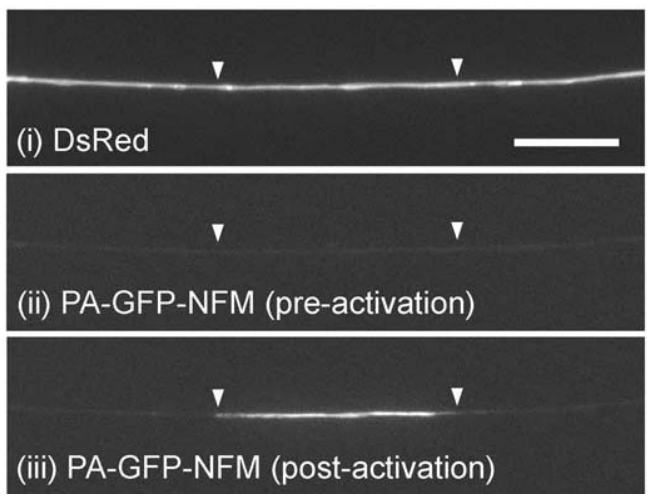

Figure 1. Characterization of PA-GFP-NFM. A, SW13 vim - cells, which lack endogenous cytoplasmic intermediate filament proteins, were cotransfected with NFL and PA-GFP-NFM constructs and allowed to express the proteins for $1 \mathrm{~d}$. This figure shows a region at the periphery of a transfected cell that was fixed and immunostained with antibodies specific for NFL (i) and NFM (ii). Single neurofilaments (arrowheads) can be resolved, because this region of the cell is very thin, and the number of neurofilaments in the cell is low. NFL and PA-GFP-NFM coassemble into neurofilaments that contain both proteins along their entire length. The diffuse fluorescence between the filaments is low, indicating that there is little unassembled protein. $\boldsymbol{B}$, Time course of activation of PA-GFP-NFM fluorescence in SW13 vim - cells coexpressing PA-GFP-NFM, NFL, and DsRed. Transfected cells were activated with 10 successive 200 ms exposures, and an image of the activated PA-GFP-NFM fluorescence was acquired after each exposure. The fluorescence intensity was quantified in each image and then normalized to the maximum activated fluorescence for that cell. This graph shows the average of the normalized data for nine cells. The optimal activation time was $1000 \mathrm{~ms}$, yielding an average of $97 \pm$ $2 \%$ (mean $\pm S D ; n=9$ ) of the maximum PA-GFP-NFM fluorescence. C, Activation of PA-GFPNFM fluorescence in cultured SCG neurons coexpressing PA-GFP-NFM and DsRed. $\boldsymbol{i}$, The DsRed fluorescence before activation. ii, The PA-GFP-NFM fluorescence before activation. The faint fluorescence in the axon is attributable to excitation cross talk caused by the broad excitation spectrum of DsRed (see Materials and Methods). iii, The PA-GFP-NFM fluorescence after activation of a $24 \mu \mathrm{m}$ region by exposure to violet light for $1000 \mathrm{~ms}$. The white arrowheads mark the boundaries of the activated region. Scale bars: $A, 5 \mu \mathrm{m} ; C, 10 \mu \mathrm{m}$.
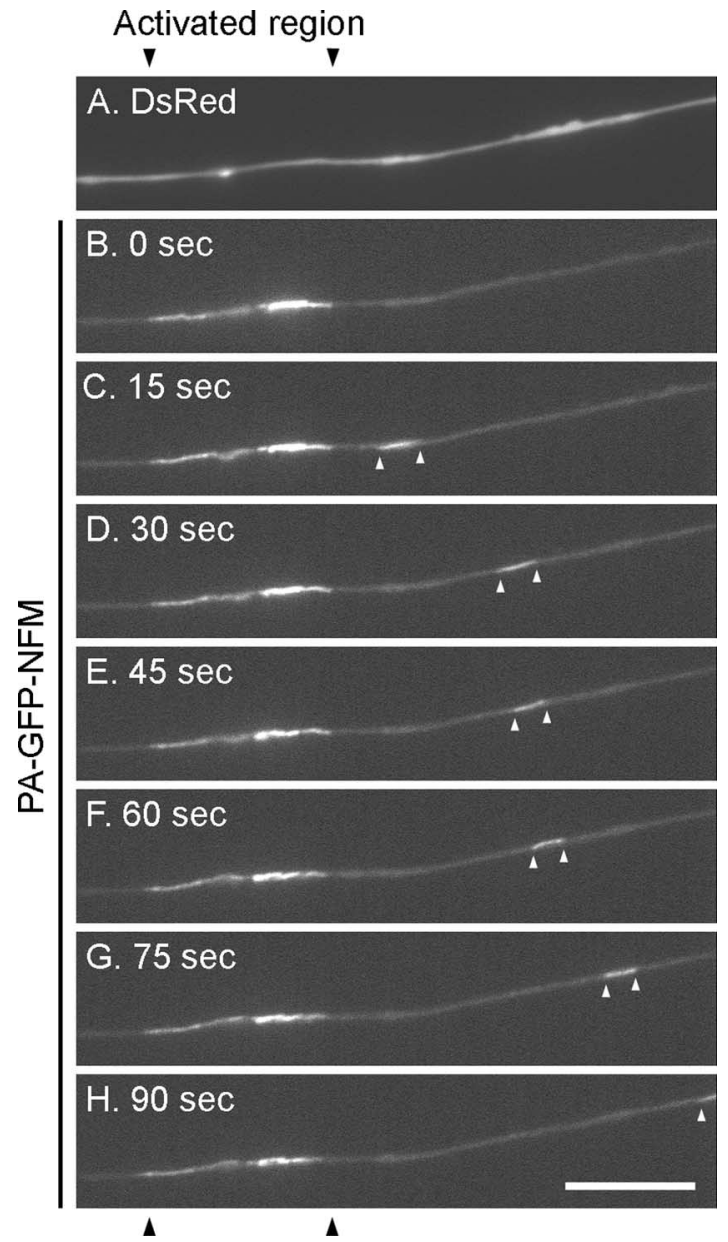

Figure 2. A neurofilament (white arrowheads) escaping from an activated region. $A, A n$ image of the DsRed fluorescence taken before photoactivation, showing the morphology of the axon. $\boldsymbol{B}-\boldsymbol{H}$, Excerpts from a time-lapse movie starting immediately after activation in the region of axon marked by the black arrowheads. Images of the activated PA-GFP-NFM fluorescence were acquired at $5 \mathrm{~s}$ intervals. This filament moved out of the activated region in an anterograde direction in the first $15 \mathrm{~s}$ after activation $(\boldsymbol{B}, \boldsymbol{C})$, paused briefly between 30 and $60 \mathrm{~s}(\boldsymbol{D}-\boldsymbol{F})$, and then resumed movement in the same direction and exited the field of view $(\boldsymbol{G}, \boldsymbol{H})$. The diffuse axonal fluorescence that can be seen outside of the activated region was present throughout the axon, even before photoactivation (see Fig. 1C), and is caused by weak excitation of the DsRed by the blue light used to excite GFP (see Materials and Methods). Proximal is left, and distal is right. Scale bar, $10 \mu \mathrm{m}$. See supplemental Movie 1 (available at www.jneurosci.org as supplemental material).

cause the region spanned the edge of a gap in the neurofilament array (Wang et al., 2000).

\section{Departure of neurofilaments from the activated regions}

To establish whether we could observe neurofilaments departing from the activated regions, we performed time-lapse imaging at $5 \mathrm{~s}$ intervals. The activated regions did not move en masse, but occasionally we observed single fluorescent filaments moving out in a rapid intermittent manner (Fig. 2, supplemental Movie 1, available at www.jneurosci.org as supplemental material). The pattern of movement was indistinguishable from what we have observed for neurofilaments moving through photobleached or naturally occurring gaps (Wang et al., 2000; Wang and Brown, 2001). In total, 10 neurofilaments departed in 25 different movies totaling $39.6 \mathrm{~min}$ of observation time (average movie length $=$ $1.6 \mathrm{~min}$ ). This corresponds to an average frequency of $\sim 0.25$ neurofilaments per minute during the first few minutes of obser- 
vation. Some of the filaments moved anterogradely, and some moved retrogradely. Occasionally the filaments exhibited brief reversals, but sustained reversals were rare. To quantify the intensity of the moving filaments, we expressed their fluorescence in terms of $\mathrm{ADU} / \mu \mathrm{m}$. The average intensity was $660 \pm 220$ $\mathrm{ADU} / \mu \mathrm{m}($ minimum $=370 \mathrm{ADU} / \mu \mathrm{m} ;$ maximum $=1300 \mathrm{ADU} /$ $\mu \mathrm{m} ; n=34)$. Assuming an average neurofilament length of 4.5 $\mu \mathrm{m}$ (Wang and Brown, 2001) and knowing the fluorescence intensities of the activated regions, we can estimate the number of neurofilaments in those regions. For the axons analyzed in the present study, the average was 21 neurofilaments per activated region $($ minimum $=5$; maximum $=154 ; n=70)$.

\section{Analysis of pulse-escape kinetics}

To investigate the pulse-escape kinetics, we acquired time-lapse movies of activated regions at $5 \mathrm{~min}$ intervals for $2 \mathrm{~h}$ (total of 25 images). To obtain finer time resolution at early times, we also acquired a second set of time-lapse movies at $40 \mathrm{~s}$ intervals for $970 \mathrm{~s}$ (total of 25 images). By attenuating the exciting light with neutral density filters (see Materials and Methods) and limiting our movies to 25 images in length, we were able to avoid measurable photobleaching. Neurofilaments departed from the activated regions infrequently and at irregular intervals, resulting in a slow and sometimes discontinuous loss of fluorescence (Fig. 3, supplemental Movie 2, available at www.jneurosci.org as supplemental material).

To analyze the rate of neurofilament departure, we quantified the change in the intensity of the fluorescence in the activated regions over time (Fig. 4). The individual axons varied considerably in their starting intensities, which reflects differences in their neurofilament content (see above). To compare the data from different axons, we normalized the fluorescence intensities in each time-lapse movie to the intensity of the activated region at the start of that movie (Fig. 5). It can be seen that there was considerable variability from one axon to the next, which is attributable to the relatively low numbers of neurofilaments in each activated region and the stochastic nature of the neurofilament movement. Remarkably, an average of $\sim 80 \%$ of the neurofilaments remained in the activated region after $15 \mathrm{~min}$ (Fig. $5 \mathrm{C}$ ), and an average of $\sim 30 \%$ remained after $2 \mathrm{~h}$ (Fig. $5 A$ ).

\section{Metabolic requirement for neurofilament transport}

To determine whether passive diffusion of neurofilament subunits contributed to the loss of fluorescence from the activated regions, we treated the cells with sodium iodoacetate, which is an inhibitor of the glycolytic enzyme glyceraldehyde-3-phosphate dehydrogenase (Sabri and Ochs, 1971). We have shown previously that sodium iodoacetate impairs neurofilament transport in cultured DRG neurons (Koehnle and Brown, 1999). In pulseescape experiments, we observed a partial reduction in the loss of fluorescence (data not shown). We then used a combination of iodoacetate and CCCP, which is a proton ionophore that uncouples oxidative phosphorylation in mitochondria and has been shown to be effective in cultured neurons (Hollenbeck et al., 1985; Miller and Sheetz, 2004). Cells treated with these two metabolic inhibitors showed no loss of fluorescence from the activated regions (Fig. 5B). Therefore, the loss of fluorescence from the activated regions was attributable to active transport, and there was no significant contribution of turnover and diffusion of fluorescent neurofilament subunits to the pulse-escape kinetics. These data demonstrate that the neurofilaments in these axons are relatively stable, and they also confirm that our imaging protocol was not associated with any measurable photobleaching.

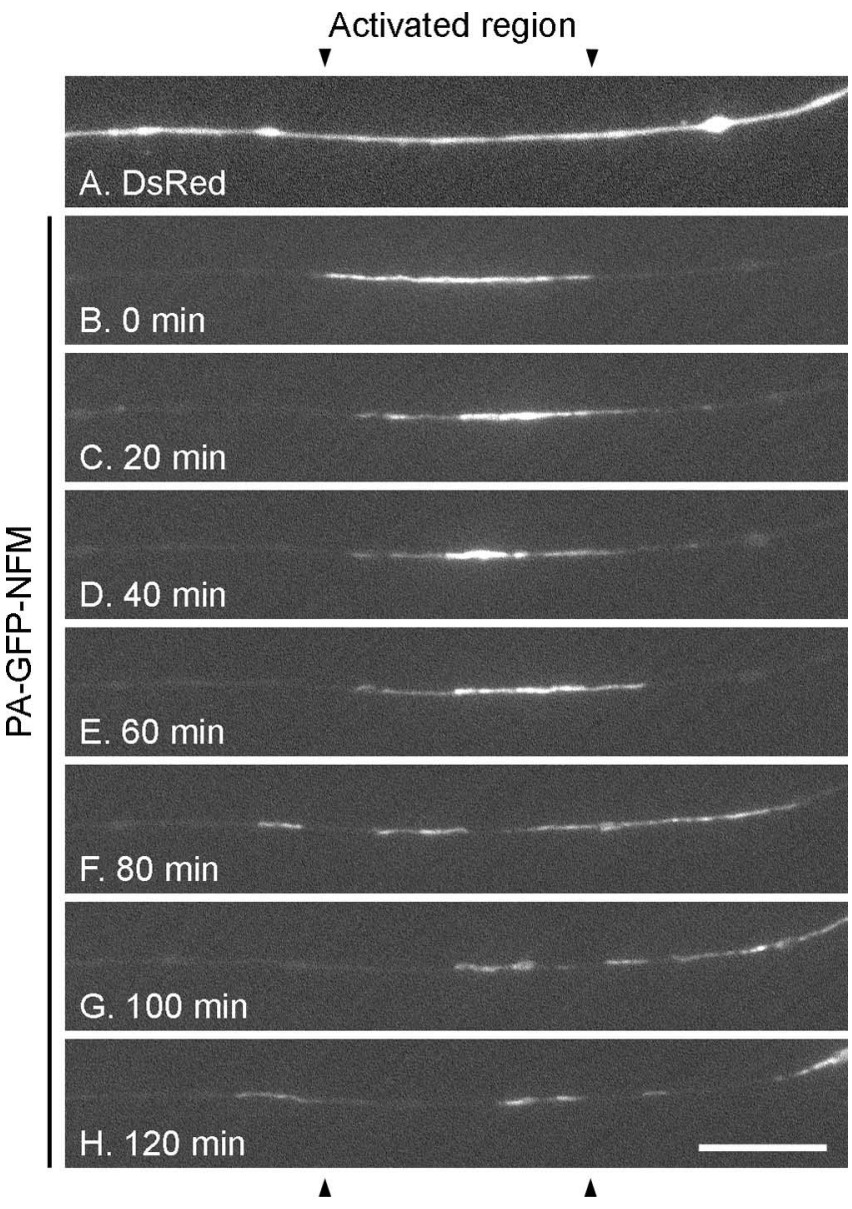

Figure 3. A pulse-escape experiment. $\boldsymbol{A}$, An image of the DsRed fluorescence taken before photoactivation, showing the morphology of the axon. $\boldsymbol{B}-\boldsymbol{H}$, Excerpts from a time-lapse movie starting immediately after activation in the region of axon marked by the black arrowheads. Images of the activated PA-GFP-NFM fluorescence were acquired at 5 min intervals for $120 \mathrm{~min}$. The loss of fluorescence was gradual and sometimes discontinuous, reflecting the small number of neurofilaments in the activated region and the stochastic nature of neurofilament movement. In this particular example, which corresponds to the graph in Figure 4D, most of the neurofilaments in the activated region moved out between 60 and 80 min after activation, but some remained after $2 \mathrm{~h}$. Individual neurofilaments cannot be tracked in these movies, because of the long duration of the time intervals. Scale bar, $10 \mu \mathrm{m}$. See supplemental Movie 2 (available at www.jneurosci.org as supplemental material).

\section{Computational modeling}

The rate of neurofilament departure from the activated regions in the pulse-escape experiments is determined by the velocity and frequency of neurofilament movement. Because the velocity of neurofilament movement is high and the frequency of neurofilament departure is low, it is clear that the pausing behavior of the neurofilaments dominates the pulse-escape kinetics for long time scales (hours rather than minutes) and that the actual velocity of neurofilament movement has little influence. For example, it takes the average moving neurofilament just tens of seconds to exit the activated region, yet $50 \%$ of the neurofilaments remained in the activated region for more than $1 \mathrm{~h}$. Thus, these data indicate that a significant proportion of the neurofilaments pause for very protracted periods in these axons.

To test this hypothesis, we simulated pulse-escape experiments using computational modeling as described in Materials and Methods. Initially, we asked what the pulse-escape kinetics would look like if the neurofilaments moved and paused in a manner identical to that observed in our previous live-cell imag- 
ing observations (Fig. 6A). To simulate this behavior, we used our published measurements of the moving and pausing behavior of neurofilaments in photobleached gaps (Wang and Brown, 2001; Brown et al., 2005). Because those observations were performed using the same neuronal cell type at the same age in culture and using identical culture conditions, we know that they accurately describe the motile behavior of the neurofilaments in our pulse-escape experiments. We found that all of the neurofilaments departed from the activated regions within $10 \mathrm{~min}$, which is much faster than we observed experimentally. This confirms that neurofilaments in these axons spend much more time pausing than is apparent from our short-term observations.

To analyze the pulse-escape kinetics, we performed curve fitting on the experimental data. We found that the kinetics were biphasic and matched a doubleexponential function, with a slow exponential decline at short times and an even slower exponential decline at longer times (Fig. 6B). The data could not be fitted with a single exponential function. This indicates that there are two distinct pausing states for neurofilaments and that these states differ in their average pause duration. Because there is no slow moving state for neurofilaments in these axons, these data indicate that there must be distinct mobile and stationary states, as predicted in our computational modeling of radioisotopic pulse-labeling experiments in vivo (Brown et al., 2005; Craciun et al., 2005). To test this hypothesis, we simulated pulse-escape experiments assuming that neurofilaments can switch between a state that is capable of movement, which we refer to as on track, and a state that is incapable of movement, which we refer to as off track. On-track neurofilaments were considered to be engaged with their cytoskeletal tracks and to exhibit the alternating movements and pauses characteristic of our previous short-term observations of moving neurofilaments. Off-track neurofilaments were considered to be temporarily disengaged from their tracks and to pause for protracted periods. Transitions between the on-track and off-track states in the model were governed by the on-track and off-track rate constants, $k_{\text {on }}$ and $k_{\text {off }}$.

As expected, $k_{\text {on }}$ and $k_{\text {off }}$ had antagonistic influences on the pulse-escape kinetics, because $k_{\text {on }}$ dictates the probability of becoming on track, and $k_{\text {off }}$ dictates the probability of becoming off track. By systematically varying $k_{\text {on }}$ and $k_{\text {off }}$, we were able to match the experimental data. Figure $6 C$ shows the best-fit simulation, which was obtained by assuming $k_{\text {on }}=0.0013$ and $k_{\text {off }}=$ 0.021 . This simulation was performed with a large number of neurofilaments (10,000 in the activated region) to minimize variment movement.
5 minute intervals
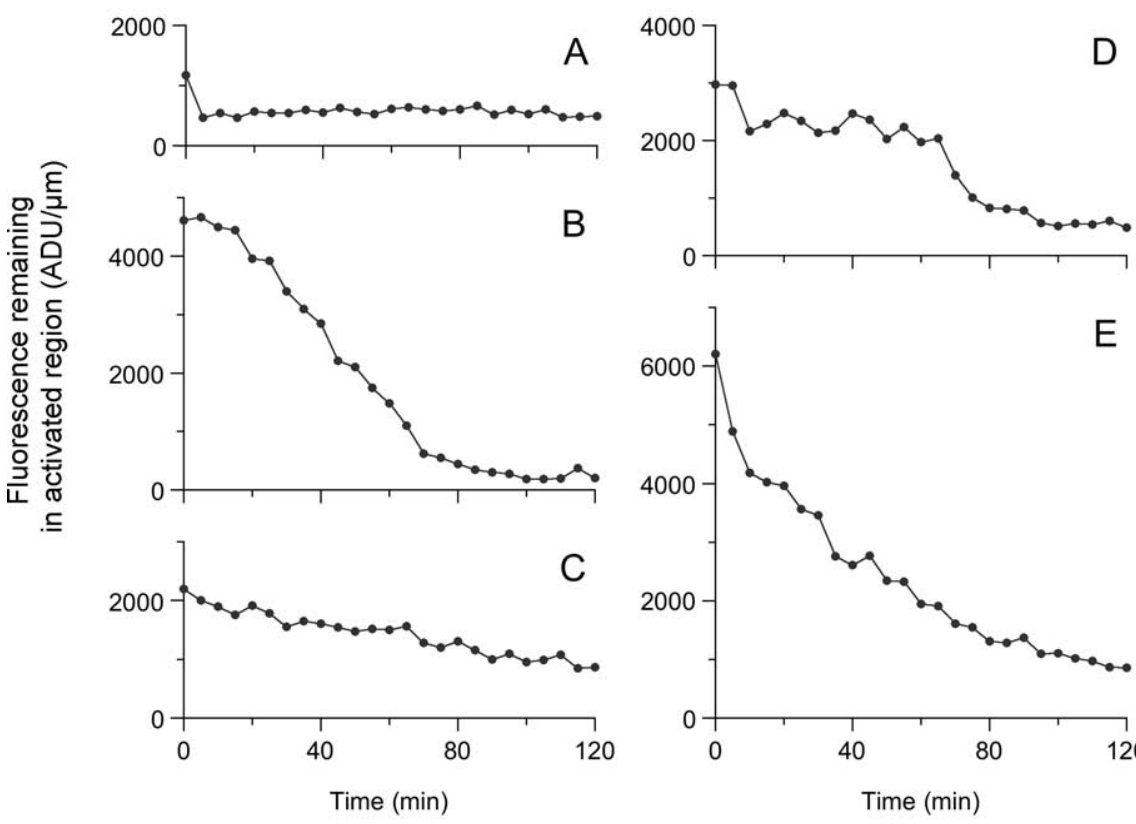

$\mathrm{E}$

40 second intervals
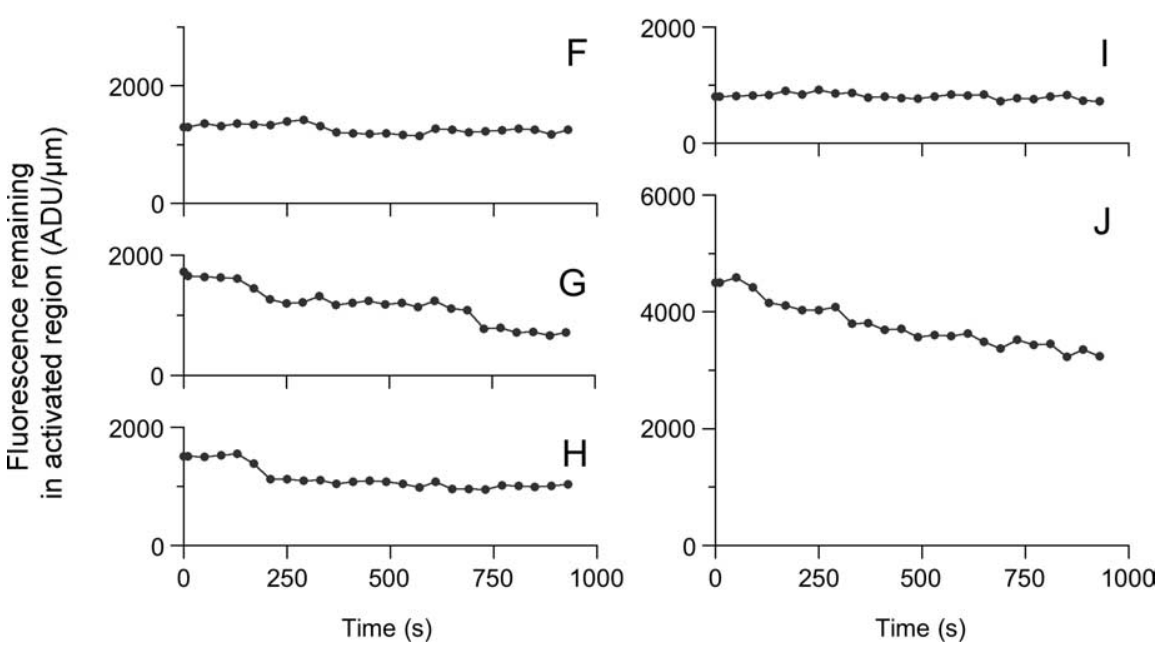

Figure 4. Pulse-escape kinetics. To analyze the pulse-escape kinetics, the fluorescence intensity in the activated regions was quantified in each frame of the time-lapse movies and expressed as ADU/ $\mu \mathrm{m} . \boldsymbol{A}-\boldsymbol{E}$, Pulse-escape kinetics for five axons that were observed for $2 \mathrm{~h}$ using $5 \mathrm{~min}$ time intervals. $\boldsymbol{F}$-J, Pulse-escape kinetics for five axons that were observed for $970 \mathrm{~s}$ using $40 \mathrm{~s}$ intervals (except for the first time interval, which was $10 \mathrm{~s}$ ). The variation in the starting intensities reflects variation in neurofilament content among the axons. Some axons showed no loss of fluorescence in the first $970 \mathrm{~s}$. All axons showed at least some loss of fluorescence after $2 \mathrm{~h}$, but the decrease was slow and sometimes discontinuous, reflecting the stochastic nature of neurofila-

ability caused by stochastic fluctuations. The number of filaments tracked in our experiments, however, was much smaller and therefore subject to significant stochastic fluctuations. To examine how well our best-fit simulation matched the experimental data, we performed 40 independent simulations with a much smaller number of neurofilaments (1000 in the activated region) and calculated the minimum and maximum values at each time point for all of the runs (Fig. $6 C$ ). The experimental data fell within the range of the stochastic fluctuations at all time points, confirming that our best-fit simulation is a good approximation of the actual behavior.

Figure $6 D-F$ shows how the best fit was identified and its 
A. 5 min intervals

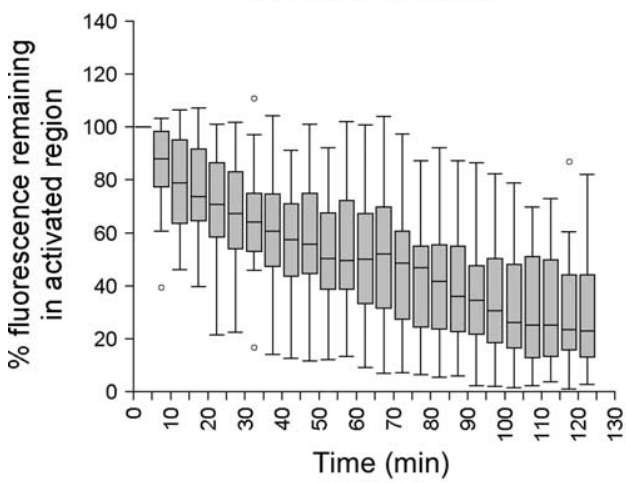

C. 40 sec intervals

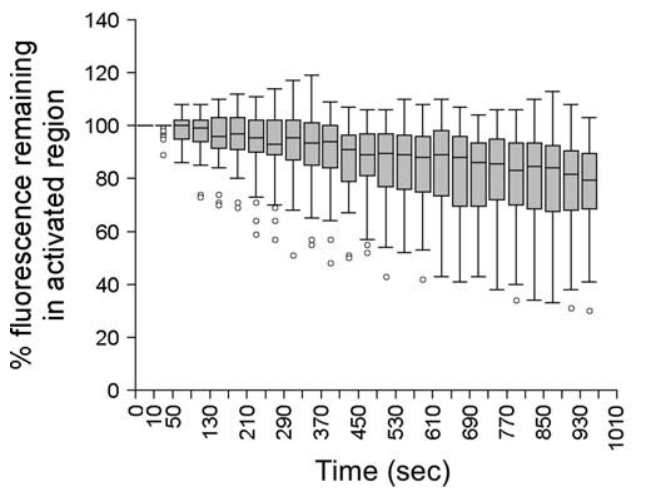

B. 5 min intervals with inhibitors
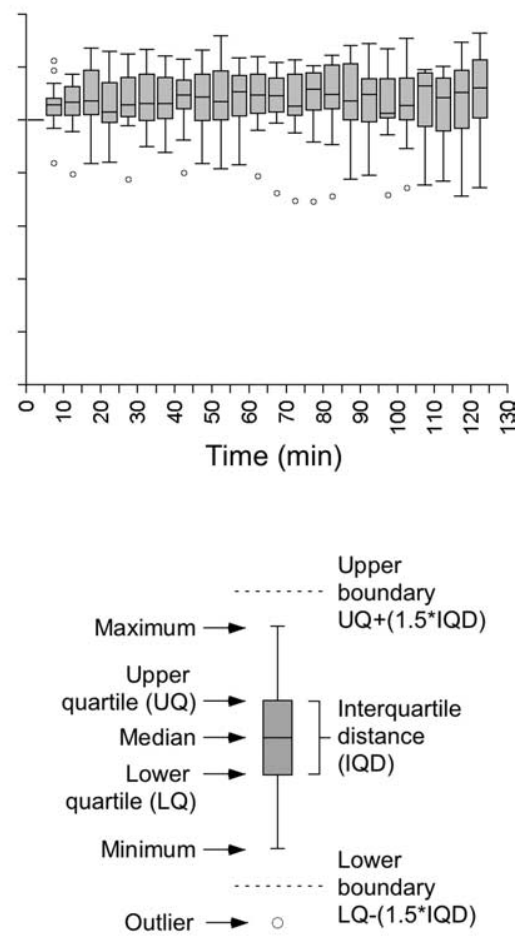

Figure 5. Box-and-whisker plots of the pulse-escape kinetics. A, Box-and-whisker plot of the pulse-escape kinetics for 16 axons that were imaged for $2 \mathrm{~h}$ at $5 \mathrm{~min}$ intervals. Note that there was considerable variability, but on average, there was a steady loss of neurofilaments from the activated regions. On average, $30 \%$ of the fluorescence remained in the activated regions after $2 \mathrm{~h}$, indicating that some neurofilaments paused for very protracted periods in these axons. $\boldsymbol{B}$, Box-and-whisker plot of the pulseescape kinetics for 16 axons that were imaged for $2 \mathrm{~h}$ at 5 min intervals in the presence of $5 \mu \mathrm{m} \mathrm{CCCP}$ and 2 mm iodoacetate. On average, there was no loss of fluorescence from the activated regions, indicating that the escape of neurofilaments from the activated region was an active process and that there was negligible loss of neurofilament from the activated regions as a result of passive diffusion and that there was no detectable photobleaching. C, Box-and-whisker plot of the pulse-escape kinetics for 52 axons that were imaged for $970 \mathrm{~s}$ at 40 s intervals (except for the first time interval, which was $10 \mathrm{~s}$ ). The key on the lower right explains the format of the box-and-whisker plots.

sensitivity to $k_{\text {on }}$ and $k_{\text {off }}$. For $k_{\text {on }}<0.0013$ or $k_{\text {off }}>0.021$, the neurofilaments spent more time off track, resulting in a slower decay than observed experimentally, whereas for $k_{\text {on }}>0.0013$ or $k_{\text {off }}<0.021$, the neurofilaments spent more time on track and escaped more rapidly than observed experimentally (Fig. $6 D, E$ ). Varying $k_{\text {on }}$ and $k_{\text {off }}$ together, keeping the ratio constant at $k_{\text {on }} / k_{\text {off }}$ $=0.0619$ (i.e., $0.0013 / 0.021$ ), had little effect on the slope at very early times $(<2 \mathrm{~min})$, because the early kinetics are dominated by neurofilaments that are already on track at the time of activation, and the proportion of on-track neurofilaments is fixed by the ratio $k_{\mathrm{on}} / k_{\mathrm{off}}$ (Fig. $6 \mathrm{~F}$ ). At later times, we observed a substantial effect on the slope, because the magnitude of $k_{\text {on }}$ and $k_{\text {off }}$ affects the frequency of cycling on and off track, and this in turn determines the duration of the off-track pauses, which dominate the long-term behavior. Reversals had little effect on the simulations, because they are so rare. Thus, our model can match the experimental data, and the values of $k_{\text {on }}=0.0013$ and $k_{\text {off }}=0.021$ make the best fit.

By tracking the behavior of the neurofilaments in our best-fit simulation, we were able to calculate their pause durations and overall transport rate. On average, the filaments spent $\sim 92 \%$ of the time off track and $\sim 8 \%$ of the time on track. Because the on-track neurofilaments in our model pause for $\sim 70 \%$ of the time (Brown et al., 2005), this means that the overall average time spent pausing (i.e., on and off track) was $\sim 97 \%$. The average on-track pause duration was $29 \mathrm{~s}$, and the average off-track pause duration was $61 \mathrm{~min}$. The average time that a neurofilament remained on track before switching off track was 5.4 min. Our previous short-term observations on neurofilaments in cultured SCG neurons have indicated that their net average velocity is $\sim 0.2 \mu \mathrm{m} / \mathrm{s}$ (excluding pauses and considering anterograde movements as positive and retrograde movements as negative) (Wang et al., 2000; Wang and Brown, 2001). Now that we know the overall average time that the neurofilaments spend pausing, we can estimate that their average transport rate in these neurons is $\sim 0.5 \mathrm{~mm} / \mathrm{d}$.

\section{Discussion}

The goal of this study was to test the hypothesis that there are distinct mobile and stationary states for neurofilaments in axons. To do this, we created a photoactivatable fluorescent neurofilament protein by fusing a photoactivatable variant of GFP to the $\mathrm{N}$ terminus of neurofilament protein $M$. The resulting PA-GFP-NFM fusion protein coassembled fully with neurofilament protein L in SW13 vim - cells, indicating that the PA-GFP domain did not interfere with assembly. To analyze neurofilament pausing behavior, we expressed the fusion protein in cultured sympathetic neurons. The fusion protein became fully incorporated into the endogenous neurofilament array, with little diffusible pool. To track neurofilament behavior, we photoactivated the fusion protein in short segments of axon by exposure to violet light. The activated protein had stability and brightness similar to conventional GFP fusions. By time-lapse imaging of the activated regions, we observed single green fluorescent neurofilament polymers moving rapidly and intermittently out of the activated regions in both anterograde and retrograde directions. We call this method the pulse-escape technique to distinguish it from our previous approaches, in which we observed neurofilament movement in naturally occurring or photobleached gaps (Wang et al., 2000; Wang and Brown, 2001).

Although the pulse-escape technique can be used to track the movement of single neurofilaments on a time scale of seconds or minutes, its real power comes not from tracking the individual neurofilaments that depart from the activated regions but rather from tracking the proportion that remains. In this way, we can observe the activated regions at intervals of minutes instead of seconds, and this permits us to observe the population behavior of neurofilaments on a time scale of several hours without measurable photobleaching. Using this approach, we found that the rate of neurofilament departure was much too slow to be accounted for by the short-term moving and pausing behavior that we have documented previously. To explain the pulse-escape kinetics, it was necessary to assume that the neurofilaments can switch between distinct mobile and stationary states, which we 

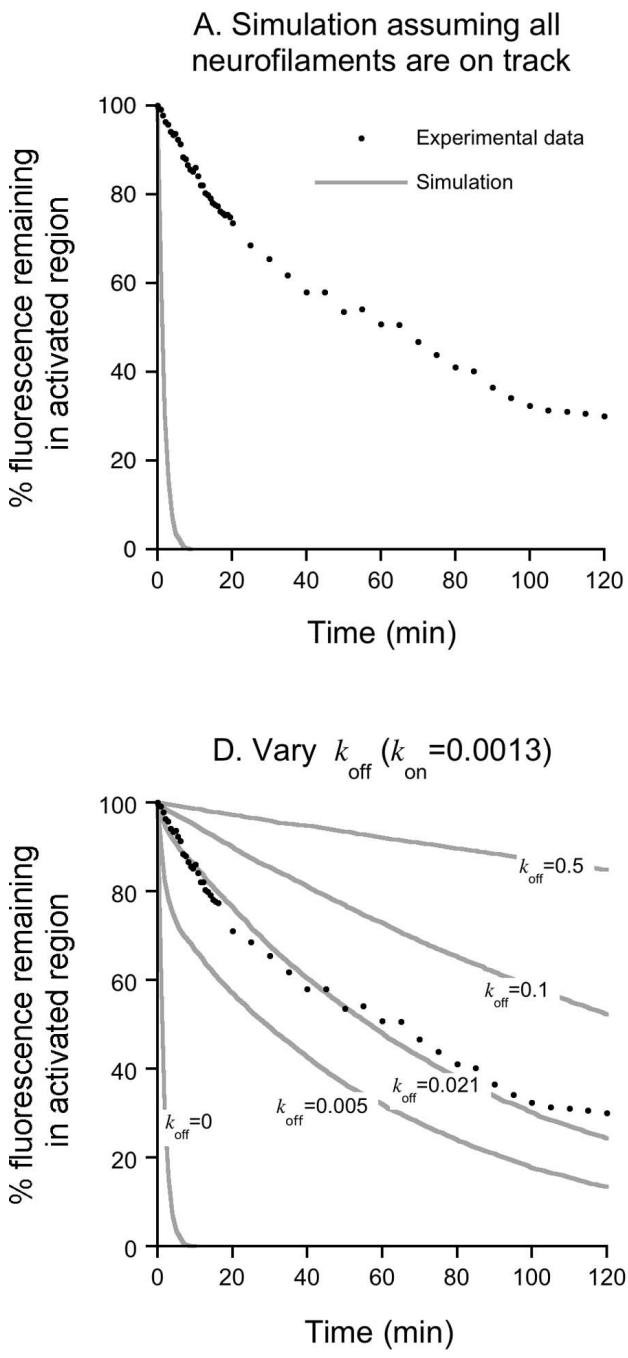
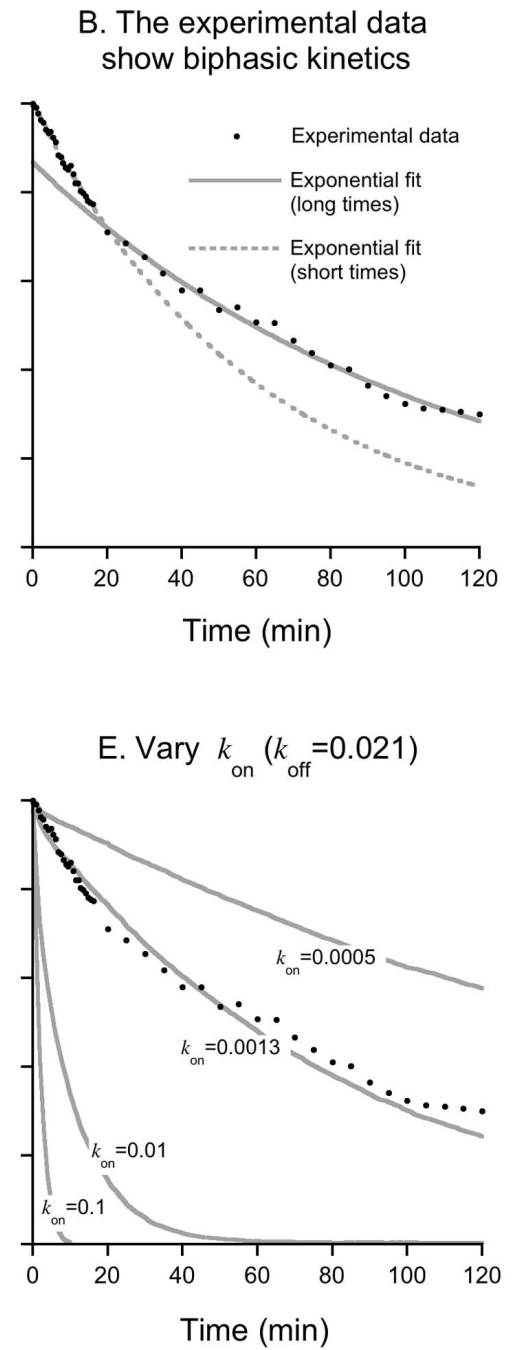

E. Vary $k_{\text {on }}\left(k_{\text {off }}=0.021\right)$

\section{C. "Best fit" simulation assuming on and off track states}

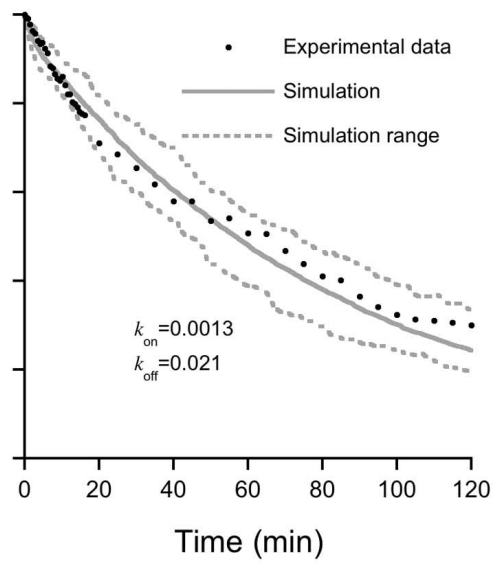

F. Vary $k_{\text {on }}\left(k_{\text {on }} / k_{\text {off }}=0.0619\right)$

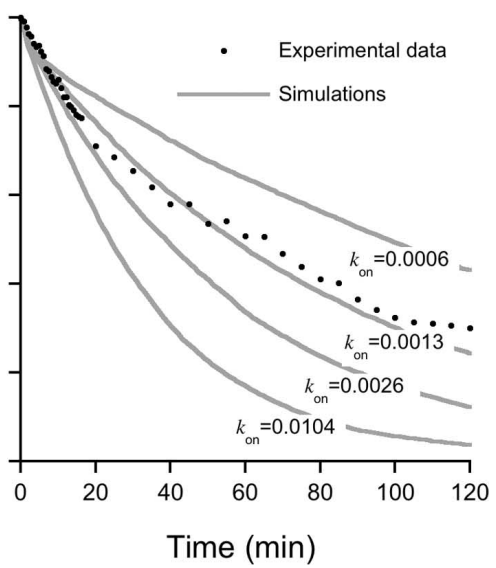

Figure 6. The pulse-escape kinetics reveal two distinct pausing states for neurofilaments. Comparison of experimental and simulated pulse-escape kinetics. The experimental data for 0 -20 min are the average of the 52 axons imaged at 40 s intervals (Fig. 5C), and the experimental data for $20-120$ min are the average of the 16 axons imaged at 5 min intervals (Fig. 5A). $A$, A simulation assuming that the neurofilaments are always on track (10,000 neurofilaments in the activated region). All neurofilaments departed within 10 min, which is much faster than the experimental data. $B$, Double-exponential curve fit of the experimental data. The decay rate at short times $(0-20 \mathrm{~min})$ is fitted with a decay constant of $0.017 \mathrm{~min}^{-1}$ (dashed line), whereas at long times ( $20-120 \mathrm{~min}$ ), it is fitted with a decay constant of $0.01 \mathrm{~min}^{-1}$ (solid line). C, Optimized "best-fit" simulation assuming that neurofilaments can be either on track or off track $\left(k_{\text {on }}=0.0013 ; k_{\text {off }}=0.021 ; 10,000\right.$ neurofilaments in the activated region). The dashed lines show the range of values obtained from 40 statistically independent simulations, each with 1000 neurofilaments in the activated region. The upper line represents the maximum value obtained, and the lower line represents the minimum. $\boldsymbol{D}$, Effect of varying $k_{\text {off }}$ on the pulse-escape kinetics (keeping $k_{\text {on }}=0.0013 ; 10,000$ neurofilaments in the activated region). Note that the simulation with $k_{\text {off }}=0$ is equivalent to assuming that the neurofilaments spend all of their time on track. $E$, Effect of varying $k_{\text {on }}$ on the pulse-escape kinetics (keeping $k_{\text {off }}=0.021 ; 10,000$ neurofilaments in the activated region). $\boldsymbol{F}$, Effect of varying $k_{\text {on }}$ and $k_{\text {off }}$ keeping the ratio $k_{\text {on }} / k_{\text {off }}$ constant $(0.0013 / 0.021=0.0619 ; 10,000$ neurofilaments in the activated region).

refer to as on and off track. Neurofilaments in the mobile state exhibit rapid bidirectional movements interrupted by short pauses characteristic of the short-term behavior that we have documented previously, whereas neurofilaments in the stationary state pause for long periods of time without any movement.

By tracking neurofilament behavior in our best-fit simulation, we showed that the neurofilaments spent $92 \%$ of their time off track and $8 \%$ of their time on track. The on-track pauses were short, averaging $\sim 30 \mathrm{~s}$. Because the average time spent on track was $5.4 \mathrm{~min}$, this indicates that the average on-track filament underwent several bouts of movement interrupted by brief pauses before switching off track. Once off track, the neurofilaments paused without movement for a very long time, averaging $\sim 1 \mathrm{~h}$. Combining the time spent pausing on and off track, the neurofilaments paused for an average of $97 \%$ of the time, which agrees well with our predictions based on computational model- ing of radioisotopic pulse-labeling experiments in vivo (Brown et al., 2005; Craciun et al., 2005). Thus, neurofilament transport is dominated by remarkably long pauses, and this explains why the overall rate of movement is so slow.

The idea that neurofilaments may be stationary in axons for long periods of time was first proposed twenty years ago by Nixon and Logvinenko (1986) based on their analysis of radioisotopic pulse-labeling kinetics in mouse optic nerve. These authors proposed that neurofilaments can become deposited into a stationary population during their transport along axons and that this stationary population slowly exchanges with the moving population over time. Since its inception, the Nixon and Logvinenko (1986) study has attracted considerable controversy. Much of the debate has centered on technical details of the radioisotopic pulse-labeling experiments, particularly the use of onedimensional SDS-PAGE to separate the radiolabeled proteins. 
Lasek et al. (1992) have argued that some faster-moving cytosolic proteins comigrate with neurofilament proteins by onedimensional SDS-PAGE, giving the appearance of two kinetic states for neurofilaments, when in fact there is only one. Using two-dimensional gels, these authors did not detect a distinct stationary component. In response, Nixon (1998) has argued that the study by Lasek et al. (1992) did not have sufficient incorporation of radiolabel into transported protein to observe the stationary phase. The two studies also differed in the radioisotopically labeled amino acid that they used, which can affect the specific activity of neurofilament proteins relative to any comigrating cytosolic proteins. Although the controversy over neurofilament transport in optic nerve remains unresolved, our modeling studies indicate that the basic notion of Nixon and Logvinenko (1986) that neurofilaments can be stationary for long periods of time and that they can switch between distinct mobile and stationary phases is correct.

If axonal neurofilaments can enter a kinetically distinct stationary state during their transport along axons, why did we not detect this state in our previous live-cell imaging studies? The answer appears to be that our previous studies were confined to short-term observations of neurofilaments moving through naturally occurring or photobleached gaps in the neurofilament array, and thus we were only able to track neurofilaments that moved during the observation period. Neurofilaments that remained paused outside of the gaps throughout the observation period could not be tracked because they could not be resolved from their neighbors. In fact, our present data indicate that the average off-track pause duration for neurofilaments in cultured neonatal rat sympathetic neurons is $\sim 1 \mathrm{~h}$, whereas the average duration of our movies of gaps has typically been on the order of 5-15 min. Thus, the gap method for observing neurofilament transport preferentially reveals the behavior of on-track neurofilaments and greatly underestimates the long-term pausing behavior (supplemental Movie 3, available at www.jneurosci.org as supplemental material). In contrast, the pulse-escape technique described in this study permits us to track the long-term behavior of the neurofilament population on a time scale of hours, not minutes, and therefore can reveal both the on-track and off-track behavior (supplemental Movie 4, available at www.jneurosci.org as supplemental material).

As their names suggest, we speculate that the on-track and off-track states correspond to neurofilaments that differ in their proximity to, or association with, the tracks along which they move. Considerable evidence now suggests that these tracks are microtubules (Shah et al., 2000; Wagner et al., 2004; Francis et al., 2005; He et al., 2005). Thus, it is interesting to note that morphometric studies on axonal cytoskeletons in vivo have demonstrated that neurofilaments generally outnumber microtubules, at least in large myelinated axons, with estimates of the neurofilament/ microtubule ratio ranging from $7 / 1$ to $97 / 1$, depending on the neuronal cell type (Price et al., 1988; Reles and Friede, 1991). In these axons, it is clear that not all neurofilaments can be adjacent to a microtubule. Thus, it is attractive to speculate that mobile and stationary neurofilaments may differ in their proximity to their microtubule tracks: mobile neurofilaments may be capable of movement because they are adjacent to their tracks, whereas stationary neurofilaments may be incapable of movement because they are temporarily stranded some distance away (Fig. 7).

It is known that neurofilaments are space-filling structures that function to increase axonal cross-sectional area. In support of this, axons in mutant animals that lack neurofilaments or the NFM tail domain fail to attain their normal caliber (Sakaguchi et

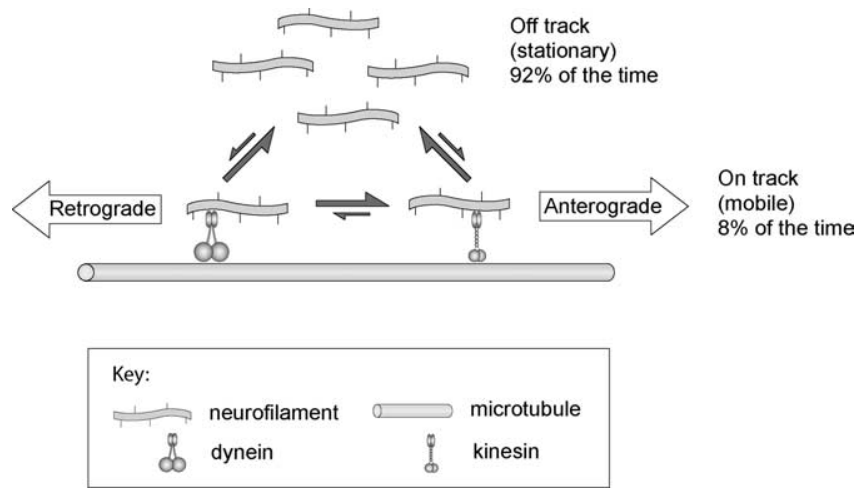

Figure 7. Distinct on-track and off-track states for neurofilaments in axons. Schematic diagram of our working model for neurofilament transport. Neurofilaments can switch between two distinct states, a mobile state, in which they are engaged with their microtubule tracks and alternate between short bouts of rapid movement and brief pauses, and a stationary state, in which they are disengaged from their microtubule tracks and pause for protracted periods without movement. We propose that the relative proportion of the time that neurofilaments spend in the on-track and off-track states is the principal determinant of their overall transport rate and distribution along axons.

al., 1993; Zhu et al., 1997; Garcia et al., 2003; Rao et al., 2003). Conversely, in diseases such as ALS and giant axonal neuropathy, neurofilaments can accumulate excessively, leading to massive balloon-like axonal swellings (Delisle and Carpenter, 1984; Donaghy et al., 1988; Sasaki et al., 1990). Thus, it is clear that axons must have mechanisms to tightly regulate their neurofilament content. One way to achieve this is to locally regulate the rate of neurofilament transport. For example, if neurofilaments enter a region of axon more rapidly than they leave, then the neurofilament content in that region will increase. In contrast, if neurofilaments leave more rapidly than they enter, the neurofilament content will decrease. Because neurofilaments switch between mobile and stationary states during their journey along the axon, any mechanism that locally recruits more neurofilaments into the mobile state will deplete neurofilaments from that region of axon, and any mechanism that recruits more neurofilaments into the stationary state will cause them to accumulate (Nixon, 1998). Thus, we speculate that the mechanisms that convert neurofilaments between their mobile and stationary states may be the principal targets of regulatory mechanisms that dictate neurofilament number and distribution along axons, and may also be the principal targets of mechanisms that lead to neurofilament misaccumulation in disease.

\section{References}

Ackerley S, Thornhill P, Grierson AJ, Brownlees J, Anderton BH, Leigh PN, Shaw CE, Miller CC (2003) Neurofilament heavy chain side arm phosphorylation regulates axonal transport of neurofilaments. J Cell Biol 161:489-495.

Brown A (2000) Slow axonal transport: stop and go traffic in the axon. Nat Rev Mol Cell Biol 1:153-156.

Brown A (2003a) Live-cell imaging of slow axonal transport in cultured neurons. In: Methods in cell biology, Vol 71, Neurons: methods and applications for the cell biologist (Hollenbeck PJ, Bamburg JR, eds), pp 305-323. Academic: New York.

Brown A (2003b) Axonal transport of membranous and nonmembranous cargoes: a unified perspective. J Cell Biol 160:817-821.

Brown A, Wang L, Jung P (2005) Stochastic simulation of neurofilament transport in axons: the "stop-and-go" hypothesis. Mol Biol Cell 16:4243-4255

Ching GY, Liem RK (1993) Assembly of type IV neuronal intermediate filaments in nonneuronal cells in the absence of preexisting cytoplasmic intermediate filaments. J Cell Biol 122:1323-1335. 
Craciun G, Brown A, Friedman A (2005) A dynamical system model of neurofilament transport in axons. J Theor Biol 237:316-322.

Delisle MB, Carpenter S (1984) Neurofibrillary axonal swellings and amyotrophic lateral sclerosis. J Neurol Sci 63:241-250.

Donaghy M, King RHM, Thomas PK, Workman JM (1988) Abnormalities of the axonal cytoskeleton in giant axonal neuropathy. J Neurocytol 17:197-208

Francis F, Roy S, Brady ST, Black MM (2005) Transport of neurofilaments in growing axons requires microtubules but not actin filaments. J Neurosci Res 79:442-450.

Garcia ML, Lobsiger CS, Shah SB, Deerinck TJ, Crum J, Young D, Ward CM, Crawford TO, Gotow T, Uchiyama Y, Ellisman MH, Calcutt NA, Cleveland DW (2003) NF-M is an essential target for the myelin-directed "outside-in" signaling cascade that mediates radial axonal growth. J Cell Biol 163:1011-1020.

He Y, Francis F, Myers KA, Yu W, Black MM, Baas PW (2005) Role of cytoplasmic dynein in the axonal transport of microtubules and neurofilaments. J Cell Biol 168:697-703.

Hollenbeck PJ, Bray D, Adams RJ (1985) Effects of the uncoupling agents FCCP and CCCP on the saltatory movements of cytoplasmic organelles. Cell Biol Int Rep 9:193-199.

Koehnle TJ, Brown A (1999) Slow axonal transport of neurofilament protein in cultured neurons. J Cell Biol 144:447-458.

Lasek RJ, Paggi P, Katz MJ (1992) Slow axonal transport mechanisms move neurofilaments relentlessly in mouse optic axons. J Cell Biol 117:607-616.

Lee MK, Xu Z, Wong PC, Cleveland DW (1993) Neurofilaments are obligate heteropolymers in vivo. J Cell Biol 122:1337-1350.

Miller KE, Sheetz MP (2004) Axonal mitochondrial transport and potential are correlated. J Cell Sci 117:2791-2804.

Nixon RA (1991) Axonal transport of cytoskeletal proteins. In: The neuronal cytoskeleton (Burgoyne RD, ed), pp 283-307. New York: Wiley.

Nixon RA (1998) Dynamic behavior and organization of cytoskeletal proteins in neurons: reconciling old and new findings. BioEssays 20:798-807.

Nixon RA, Logvinenko KB (1986) Multiple fates of newly synthesized neurofilament proteins: evidence for a stationary neurofilament network distributed non-uniformly along axons of retinal ganglion cells. J Cell Biol 102:647-659.

Patterson GH, Lippincott-Schwartz J (2002) A photoactivatable GFP for selective photolabeling of proteins and cells. Science 297:1873-1877.
Price RL, Paggi P, Lasek RJ, Katz MJ (1988) Neurofilaments are spaced randomly in the radial dimension of axons. J Neurocytol 17:55-62.

Rao MV, Campbell J, Yuan A, Kumar A, Gotow T, Uchiyama Y, Nixon RA (2003) The neurofilament middle molecular mass subunit carboxylterminal tail domains is essential for the radial growth and cytoskeletal architecture of axons but not for regulating neurofilament transport rate. J Cell Biol 163:1021-1031.

Reles A, Friede RL (1991) Axonal cytoskeleton at the nodes of Ranvier. J Neurocytol 20:450-458.

Roy S, Coffee P, Smith G, Liem RKH, Brady ST, Black MM (2000) Neurofilaments are transported rapidly but intermittently in axons: implications for slow axonal transport. J Neurosci 20:6849-6861.

Sabri MI, Ochs S (1971) Inhibition of glyceraldehyde-3-phosphate dehydrogenase in mammalian nerve by iodoacetic acid. J Neurochem 18:1509-1514

Sakaguchi T, Okada M, Kitamura T, Kawasaki K (1993) Reduced diameter and conduction velocity of myelinated fibers in the sciatic nerve of a neurofilament-deficient mutant quail. Neurosci Lett 153:65-68.

Sarria AJ, Nordeen SK, Evans RM (1990) Regulated expression of vimentin cDNA in cells in the presence and absence of a preexisting vimentin filament network. J Cell Biol 111:553-565.

Sasaki S, Maruyama S, Yamane K, Sakuma H, Takeishi M (1990) Ultrastructure of swollen proximal axons of anterior horn neurons in motor neuron disease. J Neurol 97:233-240.

Shah JV, Flanagan LA, Janmey PA, Leterrier J-F (2000) Bidirectional translocation of neurofilaments along microtubules mediated in part by dynein/dynactin. Mol Biol Cell 11:3495-3508.

Uchida A, Brown A (2004) Arrival, reversal and departure of neurofilaments at the tips of growing axons. Mol Biol Cell 15:4215-4225.

Wagner OI, Ascano J, Tokito M, Leterrier JF, Janmey PA, Holzbaur EL (2004) The interaction of neurofilaments with the microtubule motor cytoplasmic dynein. Mol Biol Cell 15:5092-5100.

Wang L, Brown A (2001) Rapid intermittent movement of axonal neurofilaments observed by fluorescence photobleaching. Mol Biol Cell 12:3257-3267.

Wang L, Ho C-L, Sun D, Liem RKH, Brown A (2000) Rapid movement of axonal neurofilaments interrupted by prolonged pauses. Nat Cell Biol 2:137-141.

Zhu Q, Couillard-Despres S, Julien J-P (1997) Delayed maturation of regenerating myelinated axons in mice lacking neurofilaments. Exp Neurol 148:299-316. 